\title{
Phase-Only Adaptive Nulling with Taguchi's Method for Antenna Array Synthesis
}

\author{
Amor Smida, Rihda Ghayoula and Ali Gharsallah \\ Department of Physics, Electronics Laboratory, Faculty of Science, Tunis El Manar 2092, Tunisia
}

Received 2012-04-20, Revised 2012-08-31; Accepted 2012-09-03

\begin{abstract}
A new and very speedy synthesis method for linear array antennas with periodic element spacing is described. This study presents an efficient method for the pattern synthesis based on Taguchi's method. A number of representative examples are presented to demonstrate the various unique capabilities of the method. A set of phase shift weights are generated in order to steer the beam towards any desired direction and avoiding interference. For isotropic linear antenna arrays parameter. The fitness function that allows the calculations of the phase shift weights is presented. The results of Taguchi's method are validated by using rectangular patch antenna and simulated by software CST2009 (CST microwave studio). Taguchi's method is briefly described in part 2. In part 3 the formulation of the array factor for linear arrays is given and fitness function will be mentioned. The numerical results optimized by Taguchi's method are given in part 4 . The last results are discuss and validated by using rectangular patch antenna and simulated by software CST2009 (CST microwave studio) in part 5. Finally, part 6 makes conclusions.
\end{abstract}

Keywords: Rectangular Patch, Radiation Pattern, Phase Shift, Array Factor, Linear Antenna Array, Synthesis Method, Antenna Arrays, Taguchi's Method

\section{INTRODUCTION}

Recently, wireless communication technologies have experienced fast growth. Spatial processing is considered the last frontier in the battle for improved cellular systems and smart antennas are emerging as the enabling technique. The use of adaptive antenna arrays in mobile handsets can facilitate the elimination of co-channel interference and multi-access interference among other problems. In fact, the synthesis of antenna arrays plays a very important role in communication systems. Actually another global optimization technique such as Taguchi's method (Taguchi et al., 2005), has been introduced to the electromagnetic and antennas communities (Weng and Choi, 2009). It was successfully used to optimize linear antenna arrays, ultra-wideband antenna, planar microwave filter design and Coplanar Waveguide (CPW) slot antenna (Lee, 2001; Ghayoula et al., 2009).

Taguchi's method is further demonstrated by optimization of linear antenna arrays. It's used to regulate phase parameter to achieve a desired angular sector and avoiding interference. This study is organized as follows.

\section{MATERIALS AND METHODS}

\subsection{Taguchi's Optimization Method}

Taguchi's optimization method will be briefly described here.

Using the concept of the Orthogonal Array (OA), (Weng et al., 2007a). Taguchi method effectively reduces the number of tests required in an optimization process. For more details, the interested reader may consult (Weng and Choi, 2009). The steps taken in Taguchi's optimization method can be illustrated in Fig. 1.

\subsection{Design of a Linear Antenna Array}

Taguchi's method is used in the synthesis of linear antenna array (Weng et al., 2007b), A set of phase shift weights are generated in order to steer the beam towards any desired direction and avoiding interference (Fig. 2) presents the antenna array geometry.

Which has $\mathrm{N}$ equally spaced elements along the axis $\mathrm{x}$. The element spacing is half-wavelength and the excitations of array elements are symmetric with respect to the axis $\mathrm{z}$ (Gie and Rahmat-Samii, 2003). Corresponding Author: Amor Smida, Department of Physics, Electronics Laboratory, Faculty of Science, Tunis El Manar 2092, Tunisia 


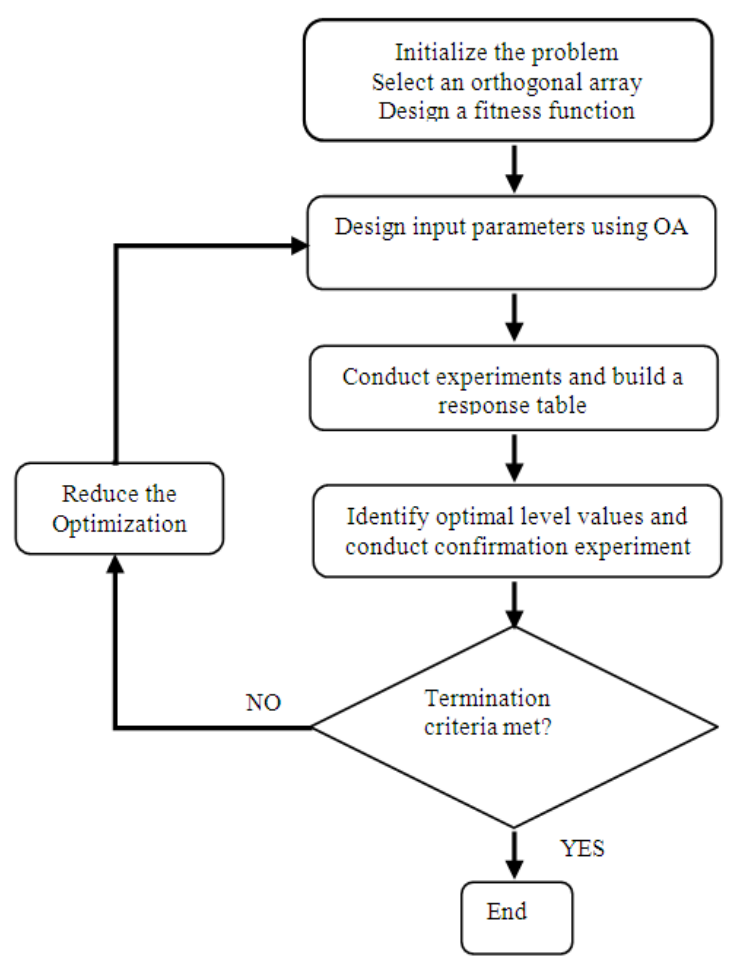

Fig. 1. Flow chart of taguchi method

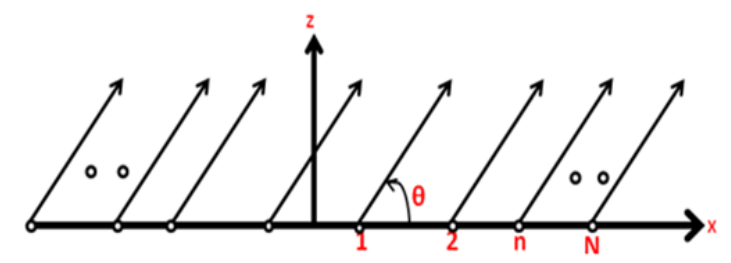

Fig. 2. Geometry of n-elements equally spaced linear array

For N-elements symmetrical array, the array factor can be written in the following Equation 1:

$$
\begin{aligned}
& \operatorname{AF}(\theta)=\sum_{n=1}^{2 N} \omega_{n} e^{j \varphi(n-1)(k d \cos (\theta)+\beta)} \\
& \psi=k d \cos (\theta)+\beta
\end{aligned}
$$

If the distance among elements is $\mathrm{d}$ and the reference point is the centre of the array, the new array factor is describes in Equation 2:

$$
\operatorname{AF}(\theta)=\sum_{n=1}^{2 N} \omega_{n} e^{\left.j \varphi(n-N-0,5) \psi+\beta_{n}\right)}
$$

Where:

$2 \mathrm{~N}=$ Number of antenna elements

$\omega_{\mathrm{n}}=$ Amplitude weight at element $\mathrm{n}$ $\beta_{\mathrm{n}}=$ Phase shift weight at element $\mathrm{n}$

$\psi=\frac{2 \pi}{\lambda} \mathrm{d} \sin (\theta)=\mathrm{kd} \sin (\theta)$

$\theta=$ Angle of interfering or desired signal

In this study, only the phase shift weights are considered, so the amplitude weights are constant. And if the phase shifts are odd symmetry, the array factor can be written in the below Equation 3 and 4:

$\mathrm{AF}(\theta)=2 \sum_{\mathrm{n}=1}^{\mathrm{N}} \cos \left[(\mathrm{n}-0.5) \mathrm{kd} \sin (\theta)+\beta_{\mathrm{n}}\right]$

And in its normalized form:

$\mathrm{AF}_{\mathrm{n}}(\theta)=\frac{1}{\mathrm{~N}} \sum_{\mathrm{n}=1}^{\mathrm{N}} \cos \left[(\mathrm{n}-0.5) \psi+\beta_{\mathrm{n}}\right]$

This equation represents a mathematical description of the antenna radiation pattern and can be used by optimization algorithms (Zuniga et al., 2010). The Taguchi algorithm is able to search for optimal phase shift weights using a fitness function based on this array factor.

\section{RESULTS}

The following example was used to demonstrate the performance of the Taguchi algorithm. We will regulate the phase parameter to achieve a desired user and avoiding interference. To resolve the problem of direct beam forming, an array of 8 Isotropic elements is defined, so $\mathrm{N}=$ 4 which is the dimension of the problem. (Fig. 3) shows an illustration of the desired and interfering angles. The geometry of the linear array is defined as follows: The distance $d$ of any two adjacent elements is set to $\lambda / 2$.

Where $\lambda$ is the wavelength. k equals to $(2 \pi) / \lambda$ and represents the wave number; amplitude $\alpha(n)=1$ and $\beta_{n}$, is the excitation phase that will be optimized in this interval $\left[-180^{\circ}, 180^{\circ}\right]$.

The algorithm stops after 100 iterations there are two conditions to be met, the fitness consists of two functions: $F$ $\left(\theta_{1}\right)$ which will attempt to maximize the value of the array factor for the direction of userl (desired angle).

While a second function, $\mathrm{F}\left(\theta_{2}\right)$ must minimize the array factor for the direction of user2 (interfering angles). The following fitness function is deduced in Equations 5-9:

fitness $=F_{1}-F_{2}$

Where:

$\mathrm{F}_{1}=\left|\mathrm{AF}\left(\theta_{1}\right)\right|^{2}$ 
$=\left|\frac{1}{N}\right|_{n=1}^{N} \cos \left[(d-0.5) \mathrm{k} \sin \left(\theta_{1}\right)+\beta_{n}\right]^{2}$

$\mathrm{F}_{2}=\left|\mathrm{AF}\left(\theta_{2}\right)\right|^{2}$

$=\left|\frac{1}{N}\right|_{n=1}^{N} \cos \left[(d-0.5) \mathrm{k} \sin \left(\theta_{2}\right)+\beta_{n}\right]^{2}$

where, $\theta_{1}$ and $\theta_{2}$ correspond to the angles of user 1 and user2 respectively.

\section{DISCUSSION}

Table 1 and 2 show the simulation results of the proposed approach when it is used with prescribed steering and null interference.

Table 1. Excitations for different steering lobes and null interference Synthesized excitations (phases)

\begin{tabular}{lll} 
& Main lobe at $-40^{\circ} \mathrm{Null}$ at $30^{\circ}$ & Main lobe at $-50^{\circ} \mathrm{Null}$ at $20^{\circ}$ \\
\hline & $\beta_{\mathrm{n}}$ & $\beta_{\mathrm{n}}$ \\
$N$ & $69.0502^{\circ}$ & $69.0538^{\circ}$ \\
1 & $162.6982^{\circ}$ & $179.0013^{\circ}$ \\
2 & $-54.3478^{\circ}$ & $-7.7110^{\circ}$ \\
3 & $44.9444^{\circ}$ & $129.8318^{\circ}$ \\
4 & $69.0502^{\circ}$ & $69.0538^{\circ}$ \\
\hline
\end{tabular}

Table 2. The different parameter of rectangular patch antenna

\begin{tabular}{lllll}
\hline $\mathrm{L}$ & $\mathrm{W}$ & $\mathrm{h}$ & $\operatorname{tg} \delta$ & $\varepsilon \mathrm{r}$ \\
\hline $36 \mathrm{~mm}$ & $36 \mathrm{~mm}$ & $4 \mathrm{~mm}$ & $2.10^{-2}$ & 2.5 \\
\hline
\end{tabular}

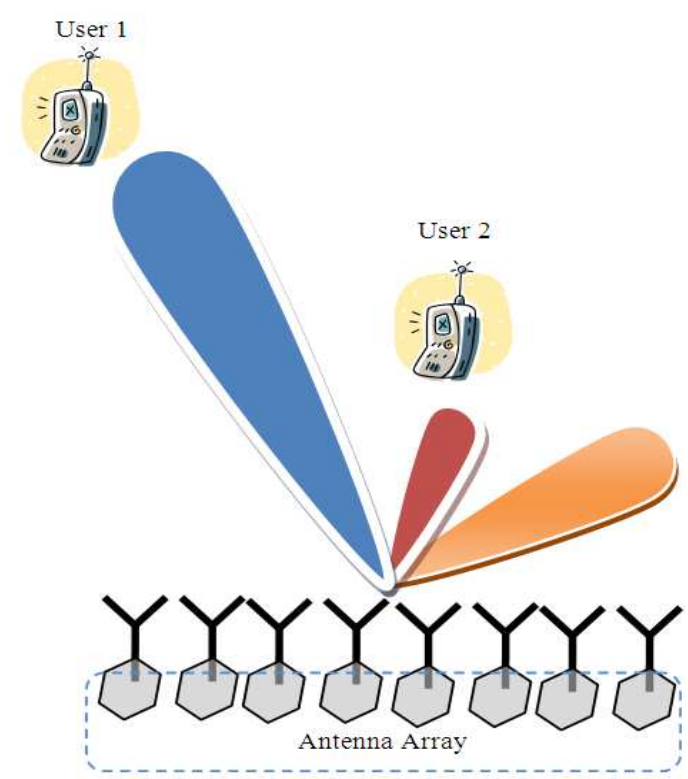

Fig. 3. Radiation pattern for desired and interfering angles
As the (Fig. 4-5) indicate, we can observe the performance of our algorithm. Figure show that the main lobe is directed to the appropriate sector and avoiding interference.

\subsection{Validation of Results Obtained by Taguchi's Method}

To validate the results obtained by our numerical tool for optimization (Taguchi's Method) we will refer to rectangular patch antenna. An eight-element linear rectangular patch (band $2.45 \mathrm{GHz}$, substrate plexy Glass with $4 \mathrm{~mm}$ height), uniformly $(\lambda / 2)$ spaced has been realised (Fig. 6) and tested for 2 cases of steered beams with null control.

The proposed design has been tested with good results. A 8-elements collinear half-wavelength patch array (Fig. 7) with centers separated is now used for synthesis purposes considering excitations with variable phases.

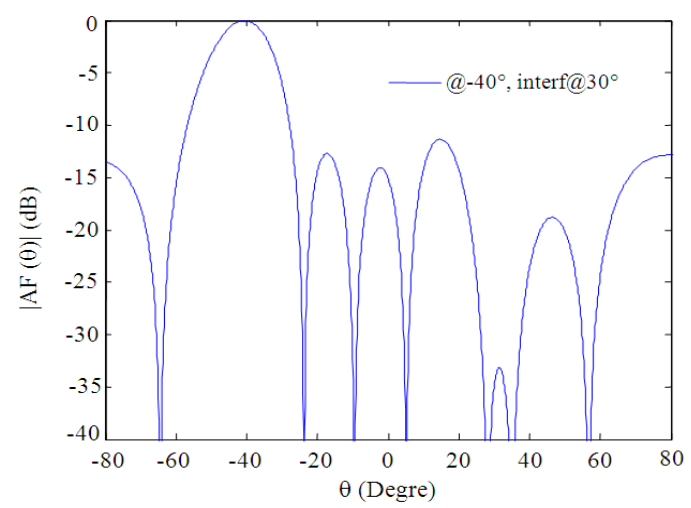

Fig. 4. Radiation pattern for desired and interfering angles $40,30^{\circ} \mathrm{C}$

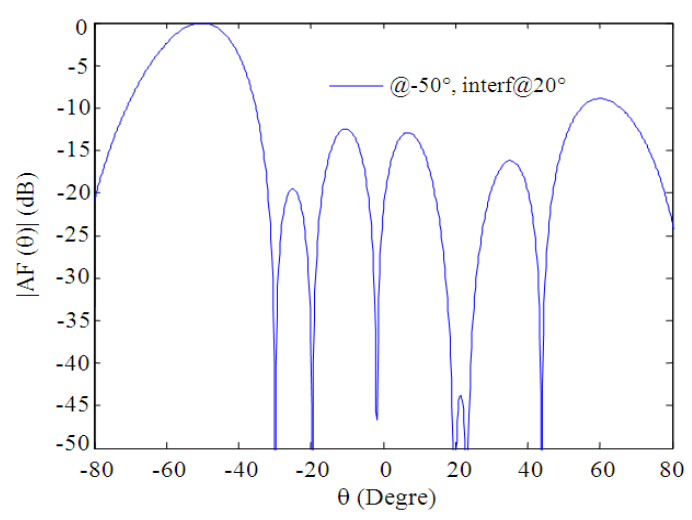

Fig. 5. Radiation pattern for desired and interfering angles $50^{\circ}, 20^{\circ}$ 
Amor Smida et al. / American Journal of Applied Sciences 9 (11) (2012) 1833-1839
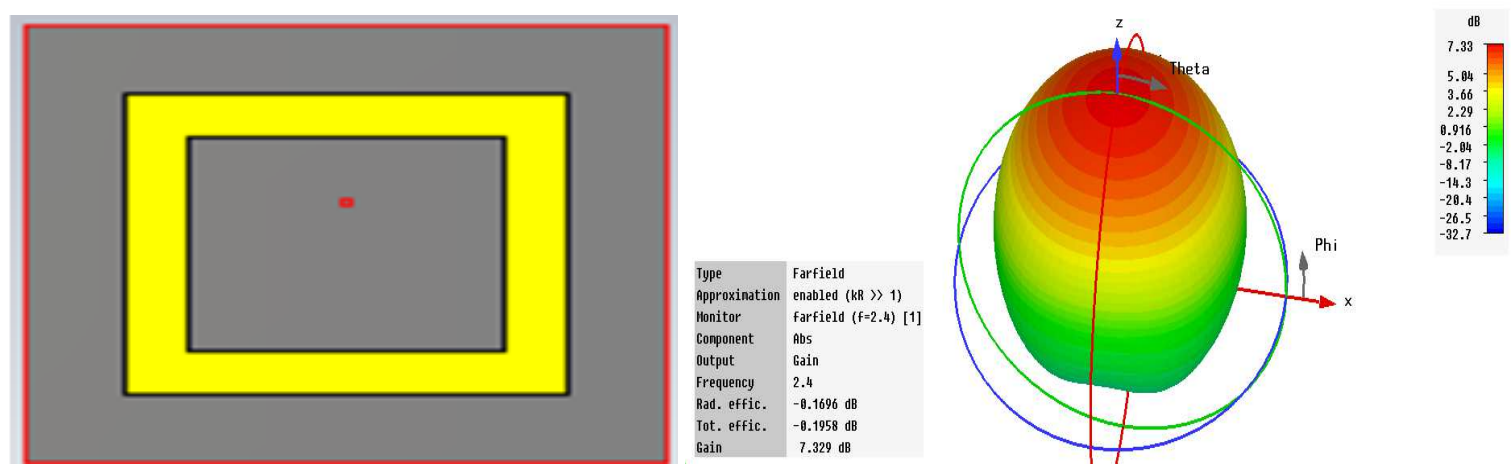

Fig. 6. The geometry of the antenna in microstrip technology (rectangular patch)

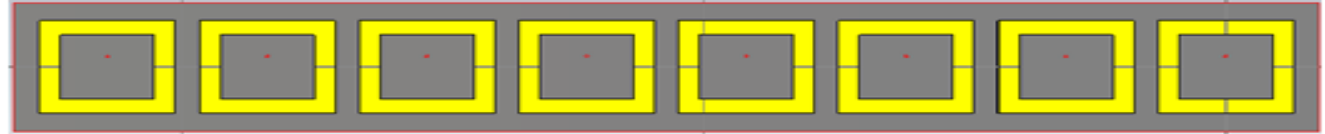

Fig. 7. Eight elements antennas array

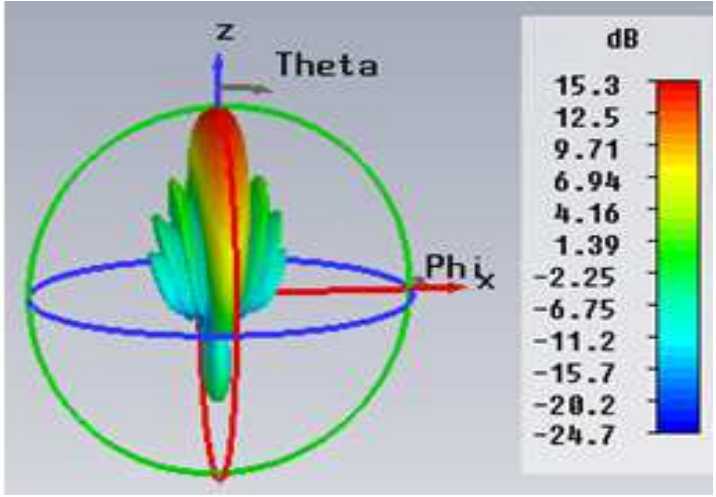

(a)

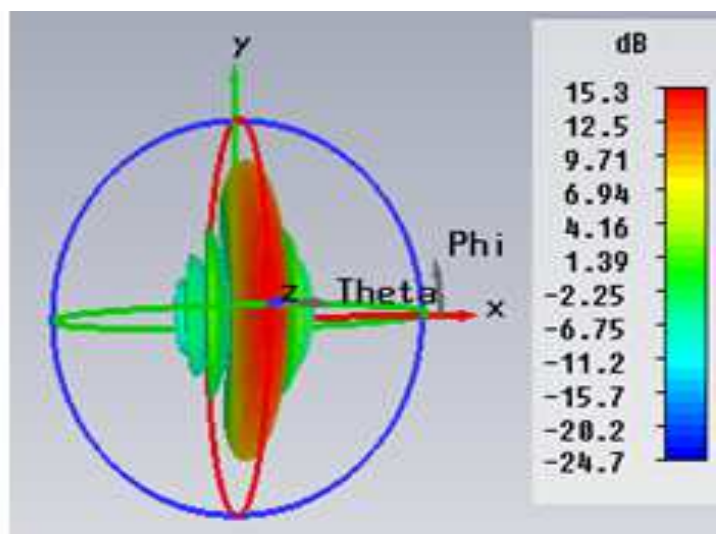

(c)

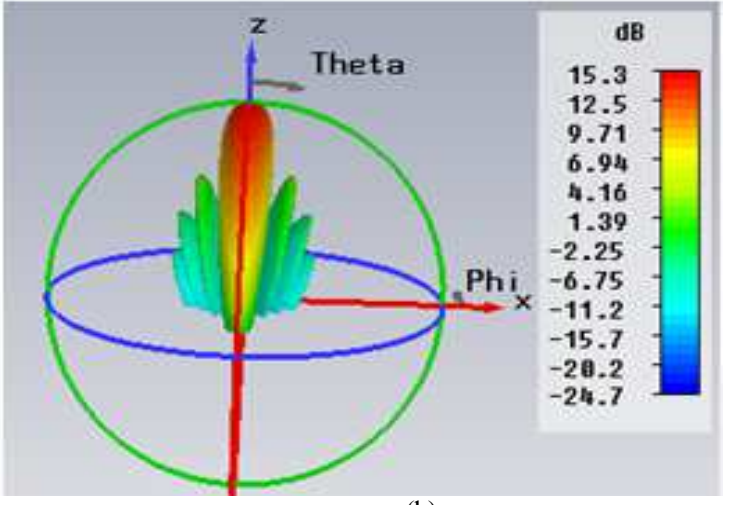

(b)

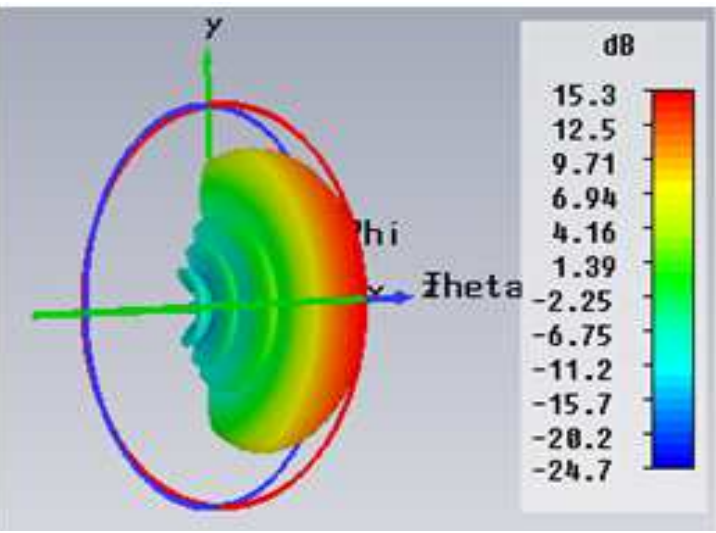

(d)

Fig. 8. (a, b, c, d). 8- Elements array pattern with (patch array antenna@2.45 GHz) 
Amor Smida et al. / American Journal of Applied Sciences 9 (11) (2012) 1833-1839

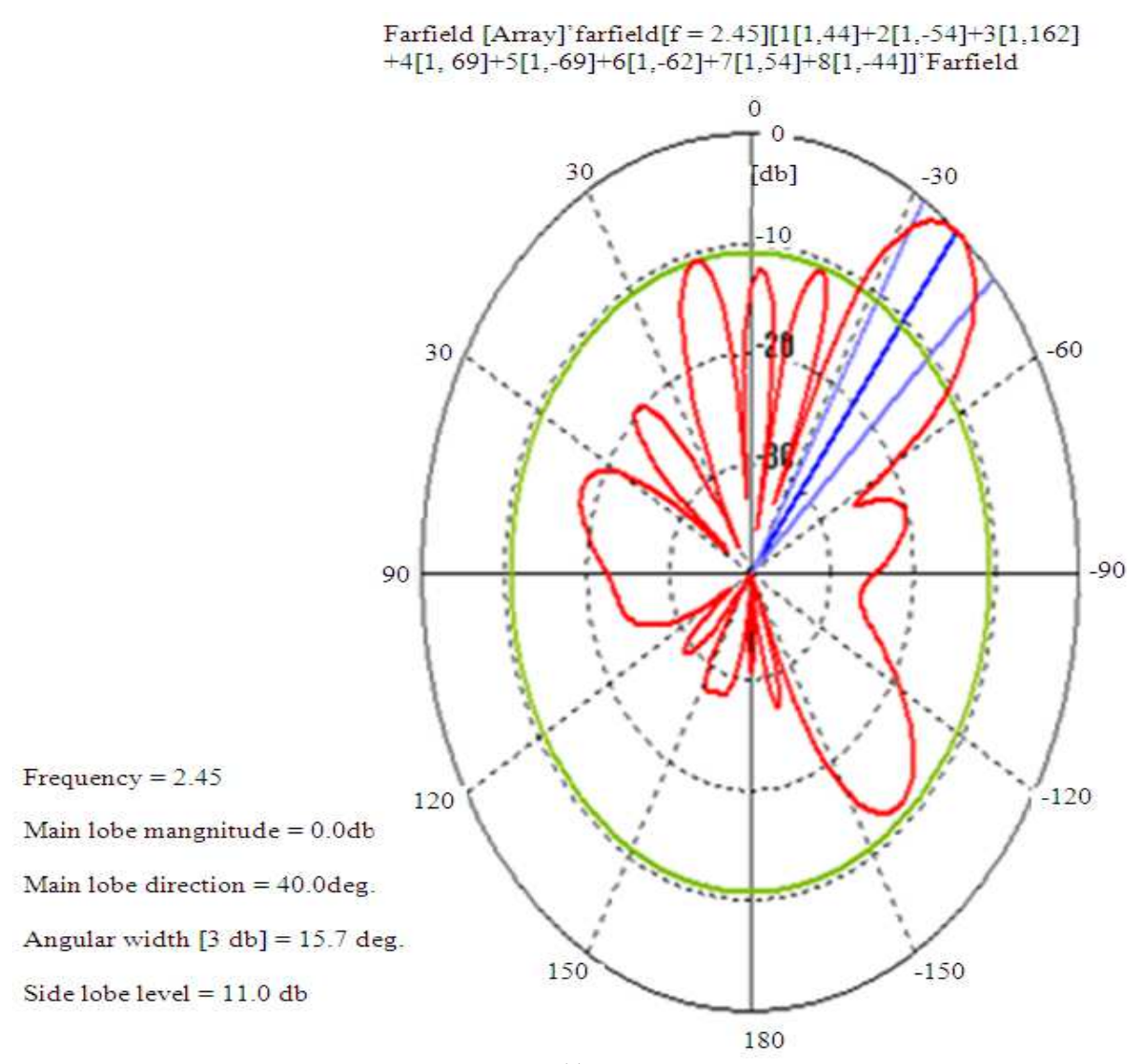

(a)

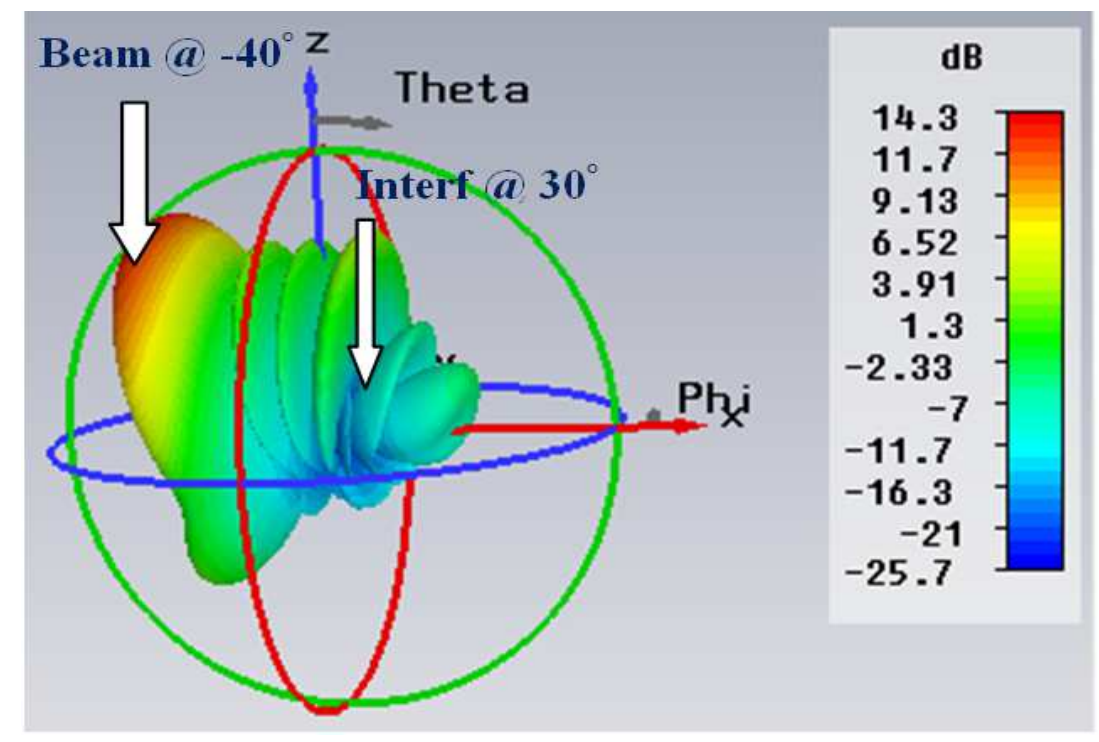

(b)

Fig. 9a, b. Radiation pattern for desired and interfering angles $-40^{\circ}, 30^{\circ}$ 
Farfield [Array]' farfield $[\mathrm{f}=2.45][1[1,44]+2[1,-54]+3[1,162]$ $+4[1,69]+5[1,-69]+6[1,-62]+7[1,54]+8[1,-44]]$ ' Farfield

Frequency $=2.45$

Main lobe mangnitude $=0.0 \mathrm{db}$ Main lobe direction $=40.0 \mathrm{deg}$. Angular width $[3 \mathrm{db}]=15.7 \mathrm{deg}$. Side lobe level $=11.0 \mathrm{db}$

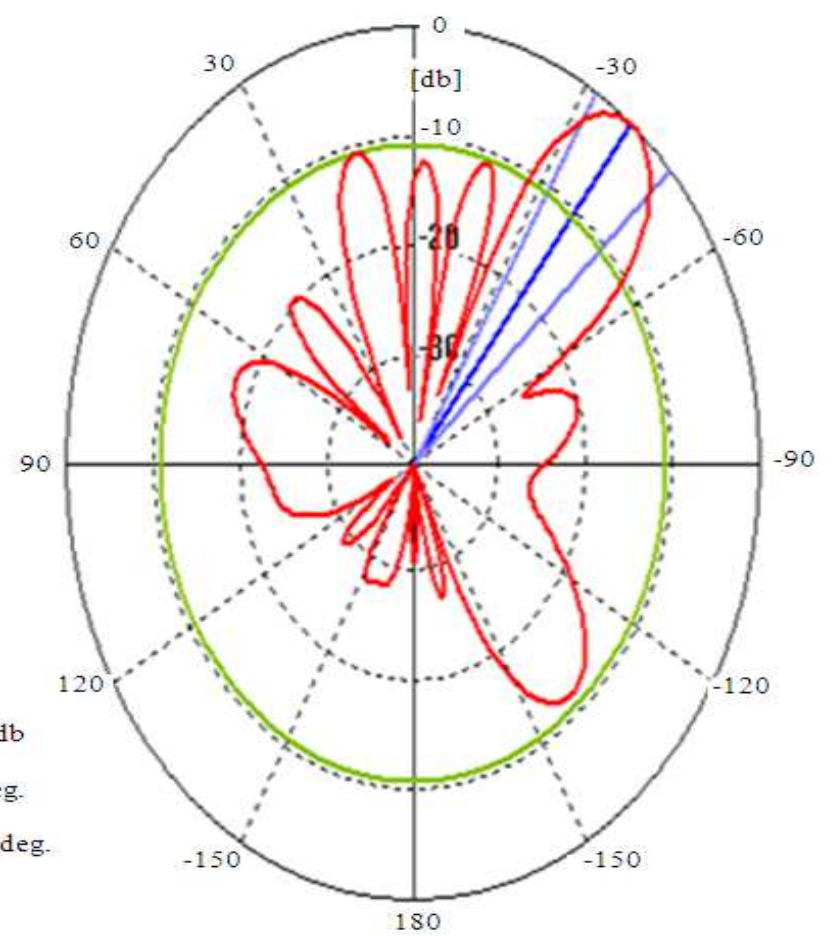

(b)

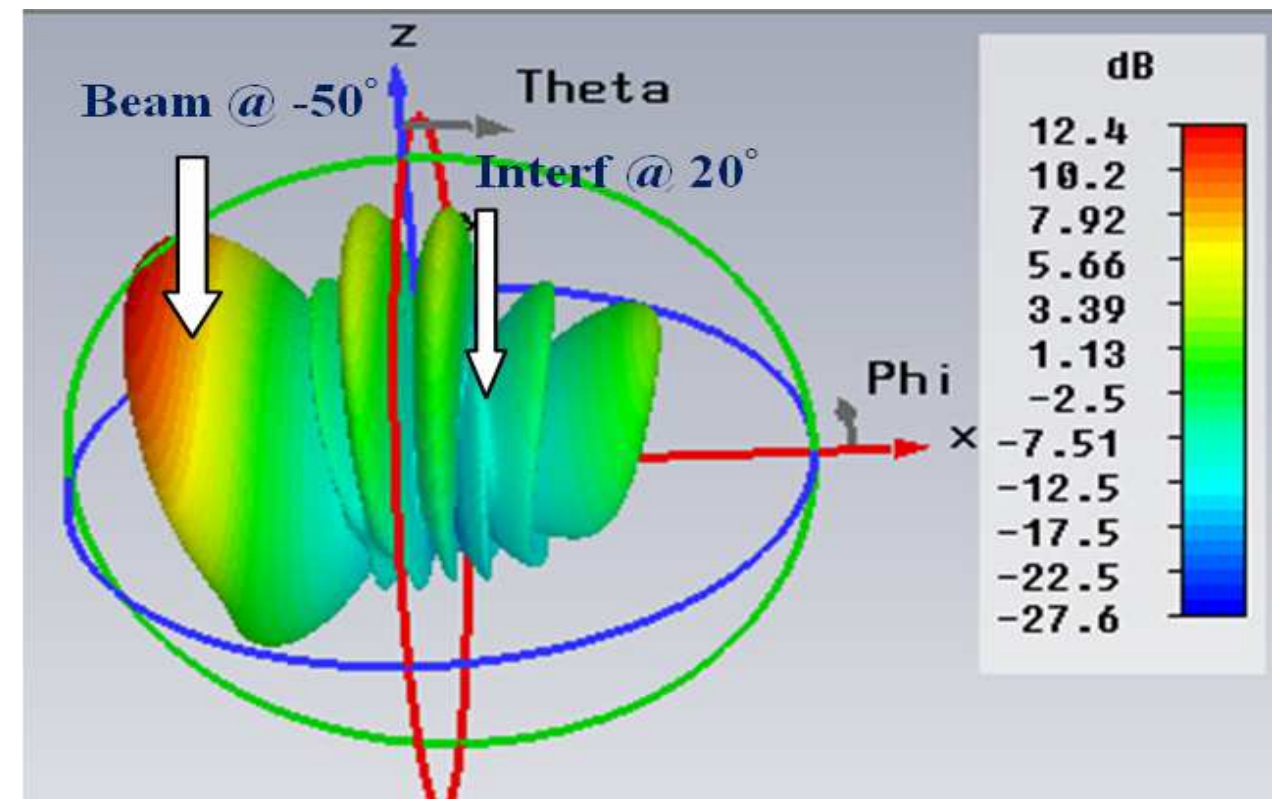

(b)

Fig. 10. (a, b) Radiation pattern for desired and interfering angles $-50^{\circ}, 20^{\circ}$ 
We have used the software CST2009 (CST microwave studio) in the simulation of radiation pattern of 8 elements linear array (rectangular patch).

Figure 8 presents the Radiation pattern simulated at different angle of view, with a frequency of $2.45 \mathrm{GHz}$ and uniform excitations (amplitude and phase).

Figure 9a, b and 10a, $b$ show radiation pattern of 8 elements linear array (patch) with excitation phases optimized by Taguchi's method (sector beam pattern and null steering).

\section{CONCLUSION}

In this study, a global optimization technique based on Taguchi's method is used (in the synthesis of linear antenna array) to obtain a set of phase shift weights. These weights were optimized in order to maximize the power of the main lobe at a desired direction while keeping nulls towards interferers. The results of Taguchi's method are validated by using array antenna (rectangular patch).At present; the possibility for optimizing both phase and amplitude is being investigated.

\section{REFERENCES}

Ghayoula, R., N. Fadlallah, A. Gharsallah and M. Rammal, 2009. Phase-only adaptive nulling with neural networks for antenna array synthesis. IET Microw. Antennas Propag., 3: 154-154. DOI: 10.1049/iet-map:20070256

Gie, D. and Y. Rahmat-Samii, 2003. Particle swarm optimization for reconfigurable phase-differentiated array design. Microw. Opt. Tech. Lett., 38: 168-175. DOI: 10.1002/mop.11005
Lee, B.G., 2001. Smart antennas: Linear array synthesis including mutual coupling effect. M.Sc Thesis, The University of Queensland.

Taguchi, G., S. Chowdhury and Y. Wu, 2005. Taguchi's Quality Engineering Handbook. Ist Edn., John Wiley and Sons, New York, ISBN-10: 0471413348, pp: 1662.

Weng, W.C. and C. Choi, 2009. Optimal design of CPW slot antennas using Taguchi's method. IEEE Trans. Magn., 45: 1542-1545. DOI: 10.1109/TMAG.2009.2012737

Weng, W.C., F. Yang and A. Elsherbini, 2007a. Electromagnetics and antenna optimization using taguchi's method. Synthesis Lectures Comput. Electromagn., 2: 1-94. DOI: 10.2200/S00083ED1V01Y200710CEM018

Weng, W.C., F. Yang and A.Z. Elsherbeni, 2007b. Linear antenna array synthesis using taguchi's method: A novel optimization technique in electromagnetics. IEEE Trans. Antennas Propagation, 55: 723-730. DOI: 10.1109/TAP.2007.891548

Zuniga, V., A.T. Erdogan, T. Arslan, 2010. Adaptive Radiation pattern optimization for antenna arrays by phase perturbations using particle swarm optimization. Proceedings of the NASA/ESA Conference on Adaptive Hardware and Systems, Jun. 15-18, IEEE Xplore Press, Anaheim, CA, USA., $\quad$ pp: 209-214. DOI: 10.1109/AHS.2010.5546256 